I fit into no category. Having completed two years of core training in hospital including a maxillofacial year, I feel I have useful skills and would like to play my part be it as a healthcare assistant or taking bloods, but thus far have drawn a blank. I haven't been able to apply for anything paid as I don't fit with computer algorithms on the online application systems and so at the minute although enjoyable I find myself staying at home unable to physically help. I have also not heard back from any volunteer roles I have applied for. When we are told things are so tough, it seems a waste for me not to be applying my skills, I was hoping there may either be more people in my unemployed predicament who have found work, or someone who knows a way in which I could help?

L. Crowder, Liverpool, UK https://doi.org/10.1038/s41415-020-1597-4

\section{Aerosol box for dentistry}

Sir, to reduce the risk of cross infection through aerosol generation we recommend a modified aerosol box design for viable dentistry.

The modified design (Figs 1a-b) comprises a reduced base width for adequate accommodation on a dental chair (without being hindered by the spittoon or arm rest). The patient end of the box is made $10 \mathrm{~cm}$ wider to accommodate wide shouldered and hefty clients. Other than two circular working ports on the doctor's end in a normal aerosol box, the dental box also has two similar working ports on the surface which is on the right side of the patient (Fig. 1c). Which is placed parallel to the U-frame base for convenient working and another single working port on the surface which is on the left side of the patient for getting assistance from that side.

The height of the box is kept at $50 \mathrm{~cm}$ to provide for adequate manoeuvrability, along with a front panel to reduce aerosol scatter in front of the patient. The extra $5 \mathrm{~cm}$ length of the frame downwards from the base U-plate will make the box more stable vertically and prevent sliding down on a tilted table (Fig. 1d). The top front $10 \mathrm{~cm}$ is attached to the posterior plate with a hinge mechanism so that it can be raised for making the patient sit up for spitting in between the procedure. Another modification to avoid the hinge mechanism is adding an arch shape to the plate over the client's neck. There is also a $2 \mathrm{~cm}$ hole on the top surface of the box to attach an aerosol suction device which can absorb the droplets from the top of the hood. It could be connected to a regular office-based suction device or a high suction aerosol suction device.

We acknowledge that the presence of the box would hinder the way a lot of procedures are performed and that there would be a learning curve to master these. However, in the wake of the current situation it would be better to not consider this device as a hindrance but more as a necessary physical infection control barrier.

B. Babu, Kerala, India, S. Gupta, V. Sahni, Chandigarh, India https://doi.org/10.1038/s41415-020-1598-3

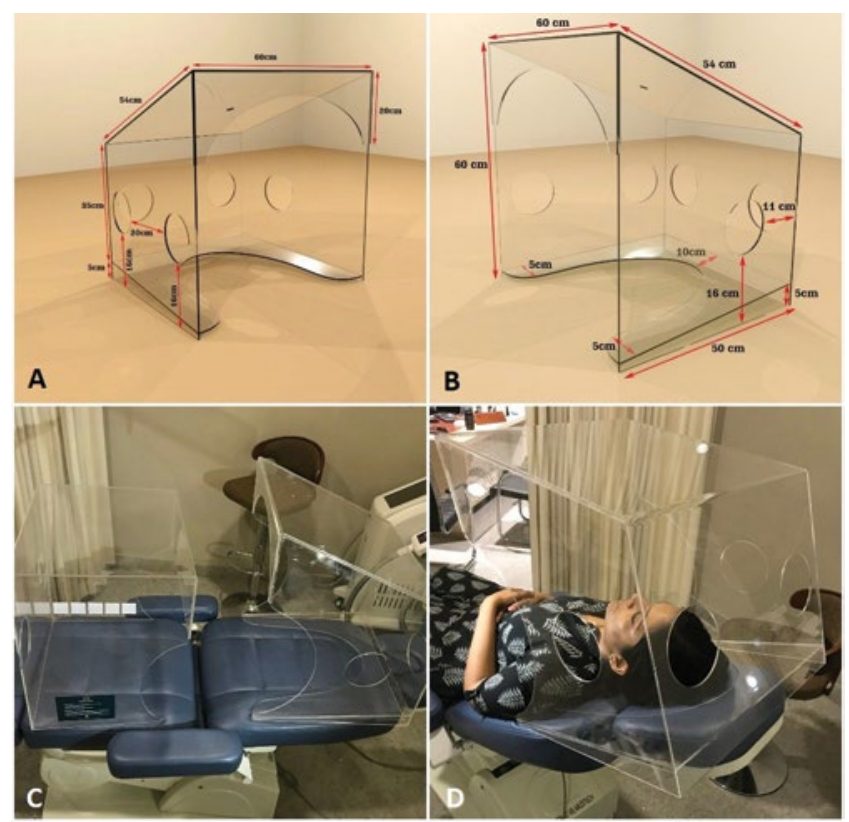

Fig. 1 Modified aerosol box design for viable dentistry

\section{Preventive dentistry}

\section{Predicting future treatment need}

Sir, the latest, thought-provoking 'big data' paper by Steve Lucarotti and Trevor Burke on patient history as a predictor of future treatment need ( $B r$ Dent J 2020; 228: 345-350) provides support for a number of not-to-be-forgotten adages in justifying the pressing need to shift to preventivelyorientated, patient-centred, minimum intervention care of patients. These include: restorations do not cure caries; the greater the need for repeat restorations, the greater the need for prevention; the only restorations which are 'permanent' are the ones you die with; once a restorative patient, always a restorative patient, and, prevention helps restorative hopes come true.

I fully concur with the view expressed by Lucarotti and Burke that '...for patients with a history of high treatment need, it is never too late to seek to switch to preventively-orientated (rather than traditional) care..., in other words saving rather than drilling away more tooth tissue. This together with the overwhelming evidence in favour of prevention in children to ideally prevent, or at least put off the day the young patient crosses the dental Rubicon and becomes a restorative patient, makes a compelling case for longitudinal, capitation care, underpinned by prevention, patient education and motivation and the adoption of modern approaches such as the repair rather than replacement of defective restorations.

In taking forward arrangements to form the College of General Dentistry (https:// cgdent.uk), one of the mantras has been, and will continue to be, the need for new, fit for future purpose approaches and standards in patient care. 'Drill and fill' must be assigned to history and replaced with 'teeth for life. Also, the dental educational continuum, including new career pathways for all members of the dental team, must be based on achieving and maintaining oral health rather than being driven by the treatment of disease.

N. Wilson, London, UK

https://doi.org/10.1038/s41415-020-1599-2 